\title{
INFLUÊNCIA DA DIETA NO DESENVOLVIMENTO E INVESTIMENTO REPRODUTIVO DE CHRYSOMYA MEGACEPHALA (FABRICIUS, 1794) (DIPTERA: CALLIPHORIDAE)
}

\section{S.M. Pires ${ }^{1}$, M.C. Cárcamo ${ }^{*}$, C.R. Zimmer ${ }^{2 * *}$, P.B. Ribeiro ${ }^{1}$}

${ }^{1}$ Universidade Federal de Pelotas, Instituto de Biologia, Departamento de Microbiologia e Parasitologia, CP 354, CEP 96010-900, Pelotas, RS, Brasil. E-mail: medpires@gmail.com

\section{RESUMO}

A dificuldade de manter-seChrysomyamegacephala em laboratório está na rápida putrefação dos meios utilizados para sua criação. Para tal, o presente trabalho visou testar sob condições controladas duas dietas: peixe in natura e um meio artificial constituído por farinha de carne, serragem e água. Foram montadas três repetições, para cada dieta, com 200 larvas cada, onde observou-se que o meio constituído por peixe in natura apresentou menor período de desenvolvimento (8 dias), emergência dos adultos de $83,3 \%$, peso médio das fêmeas fecundas de $65,185 \mathrm{mg}$, média de 220,722 ovos por fêmea, investimento reprodutivo de 3,599 e taxa de infecundidade de $43,3 \%$. O meio artificial apresentou um período maior (10 dias), a emergência dos adultos foi de $86 \%$, o peso médio das fêmeas fecundas foi de $64,882 \mathrm{mg}$, média de 218,515 ovos por fêmea, investimento reprodutivo de 3,364 e taxa de infecundidade de $24,4 \%$. Verificou-se, em ambas dietas, que houve índice de relação diretamente proporcional entre o peso e o número de ovos por fêmea, concluindo-se que a dieta influi na fecundidade das fêmeas de C. megacephala.

PALAVRAS-CHAVE: Criação em laboratório, nutrição, varejeira.

\section{ABSTRACT}

INFLUENCE OF DIET ON THE DEVELOPMENT AND REPRODUCTIVE INVESTMENT OF CHRYSOMYA MEGACEPHALA (FABRICIUS, 1794) (DIPTERA:CALLIPHORIDAE). One difficulty in the maintenance of Chrysomyamegacephala under laboratory conditions is the rapid putrefaction of the meal used as rearing media. The objective of the present study was to test under controlled conditions two different diets used as laboratory ration: raw fish meal versus an artificial medium containing meat flour, sawdust and water. The diets were tested using 200 larvae in each of three replicates. We observed that larvae reared on fish meal had an average shorter developmental period (8 days); mean adult emergence of $83.3 \%$; mean fertile female weight of $65.185 \mathrm{mg}$; mean number of eggs per female of 220.722; mean reproductive investment of 3.599; and mean infertility rate of $43.3 \%$. The larvae reared on the other artificial medium had a longer developmental period (10 days); a mean adult emergence of $86 \%$; mean fertile female weight of 64.882 ; mean number of eggs per female of 218.515; mean reproductive investment of 3.364; and mean infertility rate of $24.4 \%$. We observed that with both diets there was a proportional relation between the number and weight of eggs for each female, and therefore we concluded that diet does in fact influence the fertility of Chrysomya megacephala under laboratory conditions.

KEY WORDS: Laboratory rearing, nutrition, blowflies.

\section{INTRODUÇÃO}

Pertencente ao grupo das moscas varejeiras, Chrysomya megacephala (Fabricius,1794) (Diptera: Calliphoridae) é uma espécie exótica proveniente da Astralásia e África que foi introduzida no Brasil juntamente com Chrysomyaputoria (Wiedemann, 1830) e Chrysomya albiceps (Wiedemann, 1819) (GUIMARÃES et al., 1978), tendo seu primeiro registro no Rio Grande do Sul feito porOliveira (1982). Causadora de miíases acidentais no homem e em animais, C. megacephala possui importância médico-sanitária, pois atua como

\footnotetext{
${ }^{2}$ Universidade Federal de Pelotas, Faculdade de Agronomia “Eliseu Maciel”, Departamento de Fitossanidade, Pelotas, RS, Brasil.

*Bolsista de iniciação científica FAPERGS.

**Bolsista CAPES.
} 
importante vetor mecânico de patógenos, além de possuir alto grau de sinantropia (Costa et al., 1992).

Os imaturos deC. megacephala desenvolvem-seem substratos discretos e efêmeros (VONZUBEN et al.,2000), representados por matéria orgânica em decomposição como fezes, carcaças e vísceras (KamAL, 1958; PutMan, 1977). Esta característica confere a esta espécie importância na entomologia forense, pois auxilia na determinação do tempo de decomposição de cadáveres e, conseqüentemente, no seu intervalo pósmorte (vON ZuBEN et al., 1996).

Qualquer tipo de criação em laboratório visa reproduzir da melhor maneira possível o ambiente natural do objeto de estudo. No sudeste asiático, sua região geográfica original,C. megacephala éconhecida como a "peste do peixe seco" visto que suas larvas desenvolvem-se em peixe salgado (Esser, 1990). Para a manutenção desta espécie em ambiente controlado utiliza-se, normalmente, carne in natura, pois sabidamente este substrato faz-se atraente a adultos que o colonizam para alimentar-se e ovipositar a fim de que este sirva de recurso nutricional às suas larvas. Em laboratório, a criação de espécies com este tipo de substrato dificulta a manutenção das colônias, pois a rápida putrefação do meio, além de exalar forte odor atraindo outros artrópodes (BARBOSA et al., 2004), torna o experimento oneroso. Assim, o presente trabalho visou testar dietas alternativas, que reduzam custos, bem como adversidades causadas com o uso de meios naturais em laboratório. Para tal, a utilização do peixe como meio de criação configura a reprodução do ambiente natural da espécie em questão, enquanto que a farinha de carne, por ser um meio desidratado, caracteriza-se por ser menos perecível e de fácil conservação, justificando o referido estudo que teve como objetivos: testar uma dieta alternativa e menos perecível para criação de C. megacephala em laboratório, comparando a viabilidade e o investimento reprodutivo entre duas dietas testadas (peixeinnatura e farinha de carne).

\section{MATERIAL E MÉTODOS}

Uma colônia pré estabelecida e já adaptada de $C$. megacephala foi mantida no Laboratório de Biologia de Insetos do Departamento de Microbiologia e Parasitologia (UFPel). Os insetos foram mantidos em câmara climatizada com temperatura variando de $26^{\circ}$ $\mathrm{C} \pm 2^{\circ} \mathrm{C}$ e umidade relativa do ar acima de $75 \%$ com fotofase de \pm 12 horas. Os adultos foram criados em gaiolas teladas de dimensões $30 \times 30 \times 30 \mathrm{~cm}$ e alimentados com uma ração composta por açúcar refinado e farinha de carne em uma proporção de 2:1, além de água oferecida em copos de Becker com espuma de poliestireno cobrindo a superfície do líquido.
Para obtenção das posturas, utilizou-se um meio de cultura constituído por farinha de carne e serragem, na proporção de 3:1, sendo adicionado água até tornar o meio pastoso. Juntamente com o meio para as posturas, adicionou-se uma porção de fígado de frango a fim de proporcionar a maturidade reprodutiva das fêmeas. A renovação da colônia efetuou-se a partir de posturas da própria que foram dela transferidas para um recipiente, contendo este mesmo meio, dentro de um funil de coleta. Neste meio, após a eclosão, as larvas alimentaram-se até o terceiro ínstar, quando então o abandonaram para pupariação em um recipiente contendo serragem úmida onde foram recolhidas. As pupas foram acondicionadas em vidros telados contendo serragem moderadamente úmida e incubadas a $26^{\circ} \mathrm{C}$ até a emergência dos adultos.

Foram montadas três repetições para cada meio testado: peixe in natura e um meio artificial composto de farinha de carne sendo adicionado serragem e água até tornar-se pastoso. Foram selecionadas, aleatoriamente, 200 larvas, recém eclodidas, de primeiro ínstar de C. megacephala e colocadas em recipientes contendo $200 \mathrm{~g}$ de meio. Este recipiente foi mantido dentro de um frasco maior contendo ao fundo serragem umedecida e fechado com organza. O referido frasco, examinado diariamente a fim de acompanharse até a pupariação, foi mantido em estufa B.O.D. com temperatura de $25^{\circ} \mathrm{C}$, umidade relativa de $80 \%$ e fotofase de 12 horas. As pupas coletadas foram transferidas para vidros telados contendo serragem úmida até a emergência dos adultos.

Após a emergência, os adultos foram transferidos para gaiolas teladas recebendo alimento, além de meio, por quatro dias consecutivos, a fim de estimular-se a maturação do aparelho reprodutor. $\mathrm{O}$ alimento recebido, bem como o meio exposto para os adultos, foi o mesmo utilizado para a colônia de manutenção. Após 15 dias da emergência, 90 fêmeas de cada dieta testada foram coletadas, de forma aleatória, e anestesiadas com acetato de etila para que fossem pesadas em balança analítica e dissecadas sob estéreo-microscópio para, assim, estimar o investimento reprodutivo que segundo Collins (1980), consiste na divisão do número de ovos pelo peso fresco da fêmea.

Foram realizadas análises estatísticas a fim de avaliar-se a associação entre peso e fecundidade das fêmeas, criadas em dieta de peixe in natura e farinha de carne, bem como comparar a fecundidade das fêmeas criadas em ambas as dietas. Para tal, efetuouse o teste do Qui-quadrado onde os resultados foram considerados estatisticamente significativos quando se observou $\alpha=0,01$. Foi realizado, ainda, com o auxílio do programa STATISTIX 8 (2003), análise de variância no nível de 0,05 de significância. 


\section{RESULTADOS E DISCUSSÃO}

Os resultados obtidos demonstraram que, somando-se as três repetições (600 larvas), o meio constituído por peixe in natura apresentou viabilidade larval de $95 \%$ (570) e emergência dos adultos de 83,3\% (500). Omeio artificial, constituído de farinha de carne como fonte protéica, apresentou viabilidade larval de 91,5\% (549) e $86 \%$ (516) dos adultos emergiram. A sexagem dos adultos permitiu ainda verificar que o meio constituído de peixe resultou em 237 (47,40\%) fêmeas e 263 $(52,60 \%)$ machos, enquanto que o meio de farinha de carne resultou em 253 (49,03\%) fêmeas e 263 (50,97\%) machos, com tendência de $50 \%$ para cada sexo em ambas as dietas.

A amostra das fêmeas examinadas, provenientes de larvas alimentadas com peixe, apresentou peso variando entre $25 \mathrm{mg}$ e $86 \mathrm{mg}$ e o número de ovos por fêmea variou entre 0 e 311 . As alimentadas durante a fase larval com farinha de carne também apresentaram número de ovos por fêmea compreendido entre 0 e 311, porém tiveram um peso fresco maior cujo valor ficou entre $26 \mathrm{mg}$ e $90 \mathrm{mg}$. Trabalhos com moscas realizados anteriormente, demonstraram que o tamanho e o peso do adulto são reflexos da quantidade e da qualidade do alimento consumido durante o estágio larval (Silveira Neto, 1976; GoOdBROD;GoFF, 1990; REIs et al., 1994; ZIMMER et al., 2006).

Considerando-se apenas as fêmeas férteis, a média de peso daquelas mantidas no peixe durante a fase larvalfoide65,185mg士10,285eonúmeromédiodeovos por fêmea foi de 220,722 $\pm 71,580$. Neste caso, o investimento reprodutivo apresentou valor de 3,599 $\pm 0,838$ (Tabela 1). A análise de correlação entre o peso das fêmeas fecundas e o número de ovos por fêmea apresentou coeficiente de 0,398 . As fêmeas fecundas alimentadas com farinha de carne na fase larval apresentaram peso médio de $64,882 \mathrm{mg} \pm 9,166$ e a média do número de ovos por fêmea foi de $218,515 \pm 60,918$. O valor do investimento reprodutivo foi de 3,364 $\pm 0,861$ (Tabela 1). Ocoeficiente de correlação entre peso das fêmeas fecundas e número de ovos por fêmea foi de 0,524.

Comparando as dietas, através da análise devariância verificou-sequeos valores do peso dasfêmeasfecundas $(\mathrm{P}=0,899 ; \mathrm{F}=0,61)$ e do número de ovos por fêmea $(\mathrm{P}=0,702 ; \mathrm{F}=0,81)$ mostraram-se não significativos. Segundo PARRA (1990), é durante a fase larval que os insetos tendem a escolher umalimento apropriado para consumi-lo em proporções balanceadas de tal forma a utilizá-lo adequadamente para promover um ótimo crescimento e desenvolvimento, dando origem a um adulto que seja reprodutivamente competitivo.

Analisando as fêmeas fecundas e infecundas mantidas no peixe durante sua fase larval, o número de ovos por fêmea foi diretamente proporcional ao peso (Fig. 1), demonstrando índice de correlação de 0,692 . Das 33 fêmeas que apresentaram peso igual ou inferior a $51 \mathrm{mg}$, verificou-se que $81,81 \%$ (27) foram infecundas e o índice de fecundidade foi de apenas $18,18 \%$ (6). Já as que apresentaram peso superior a 51 mg (57), foram fecundas em 78,95\% (45) dos casos e infecundas em $21,05 \%$ (12).

Tabela 1 - Influência da farinha de carne e do peixe in natura sobre a infecundidade das fêmeas (FI), peso das fêmeas fecundas (PFF), número de ovos por fêmea (O/F) e investimento reprodutivo (IR) de Chrysomya megacephala, em laboratório.

\begin{tabular}{lcccr}
\hline Meios de cultura & FI $(\%)$ & PFF $(\mathrm{mg})$ (Média \pm DP) & O/F (Média \pm DP) & IR(Média \pm DP) \\
\hline Farinha de carne & 24,4 & $64,882 \pm 9,166$ & $218,515 \pm 60,918$ & $3,364 \pm 0,861$ \\
Peixe & 43,3 & $65,185 \pm 10,285$ & $220,722 \pm 71,580$ & $3,599 \pm 0,838$ \\
\hline
\end{tabular}

DP = Desvio Padrão

Tabela 2- Associaçãoentre peso das fêmease a fecundidade de Chrysomya megacephala, cujas larvas se desenvolveram em peixe in natura, em laboratório.

\begin{tabular}{lccc}
\hline & $\begin{array}{c}\text { Fêmeas } \\
\text { férteis }\end{array}$ & $\begin{array}{c}\text { Fêmeas } \\
\text { inférteis }\end{array}$ & \\
\cline { 2 - 3 } Peso (mg) & Freq. abs. (\%) & Freq. abs. (\%) & Total (\%) \\
\hline$\leq 51$ & $6(6,67)$ & $27(30,00)$ & $33(36,67)$ \\
$>51$ & $45(50,00)$ & $12(13,33)$ & $57(63,33)$ \\
Total & $51(56,67)$ & $39(43,33)$ & $90(100)$ \\
\hline
\end{tabular}

Freq. abs. = Freqüência absoluta
Tabela 3 - Associação entre peso dasfêmeas e a fecundidade de Chrysomya megacephala, cujas larvas se desenvolveram em farinha de carne em laboratório.

\begin{tabular}{lccc}
\hline Peso (mg) & $\begin{array}{c}\text { Fêmeas } \\
\text { férteis }\end{array}$ & $\begin{array}{c}\text { Fêmeas } \\
\text { inférteis }\end{array}$ & \\
\cline { 2 - 3 } & Freq. abs. (\%) & Freq. abs. (\%) & Total \\
\hline$\leq 51$ & $7(7,78)$ & $14(15,55)$ & $21(23,33)$ \\
$>51$ & $61(67,78)$ & $8(8,89)$ & $69(76,67)$ \\
Total & $68(75,56)$ & $22(24,44)$ & $90(100)$ \\
\hline
\end{tabular}

Freq. abs. = Freqüência absoluta 
Observou-se que houve relação direta entre peso e fecundidade quando se compara as freqüências relativas e absolutas das fêmeas fecundas e infecundas com o seu peso fresco (Tabela 2). Verificou-se que, do total das 90 fêmeas criadas em peixe in natura e cujo peso é igual ou inferior a $51 \mathrm{mg}$, apenas 6,67\% (6) foram fecundas e 30,00\% (27) infecundas. As fêmeas que apresentaram peso superior a $51 \mathrm{mg}, 50,00 \%$ (45) foram fecundas e apenas $13,33 \%$ (12) foram infecundas. A análise do $\chi^{2}$ demonstra associação entre peso e fecundidade das fêmeas com alto índice de significância $(\alpha=0,01)$ onde este apresentou valor de 49,87 , ou seja, o peso das fêmeas influi sobre a fecundidade de C. megacephala.Segundo alguns autores, a redução no tamanho e no peso das fêmeas dos Diptera produz um importante efeito na dinâmica da população visto que as fêmeas pequenas diminuem a fecundidade (vON ZuBEN, 1993; ReIs etal., 1996; TARDELLI et al., 2004).

As fêmeas fecundas e infecundas mantidas na farinha de carne também apresentaram número de ovos por fêmea diretamente proporcional ao peso (Fig. 2) demonstrando índice de correlação de 0,714. Das fêmeas que apresentaram peso igual ou inferior a $51 \mathrm{mg}$ (21), verificou-se que apenas 33,33\% (7) foram fecundas e a grande maioria, 66,66\% (14), foram infecundas. Já as que apresentaram peso superior a 51 mg (69) foram fecundas em $88,40 \%$ (61) dos casos e infecundas em $11,59 \%$ (8).

A relação direta entre peso e fecundidade pode ser observada na Tabela 3. As fêmeas que apresentaram peso igual ou inferior a $51 \mathrm{mg}$ tiveram índice de fecundidade de apenas 7,78\% (7) enquanto 15,55\% (14) foram infecundas. Já as fêmeas que tiveram peso superiora $51 \mathrm{mg}$ apresentaramfecundidadede $67,78 \%$ (61), com apenas 8,89\% (8) de infecundidade. Esses números demonstraram a relação direta das variáveis peso e fecundidade das fêmeas. Tais resultados se confirmam pelo alto índice de significância $(\alpha=$ $0,01)$ corroborando pelo teste do $\chi^{2}\left(\chi^{2}{ }_{\mathrm{c}}=27,66>\chi_{\mathrm{t}}^{2}=\right.$ 11,34).

Considerandoos dois tratamentos utilizados, peixe in natura efarinha de carne, ambos não demonstraram diferenças significativas quanto ao peso das fêmeas, o número de ovos por fêmea e, conseqüentemente o investimento reprodutivo. A dieta de peixe in natura, embora tenha proporcionado um período menor para a conclusão do ciclo (Tabela 4) e assim um menor intervalo entre as gerações, apresentou índice de infecundidade de $43,3 \%$ (39) das fêmeas examinadas.

Já o meio de farinha de carne apresentou um período maior (Tabela4), levando a um maior intervalo entre as gerações, mas apenas $24,4 \%$ (22) das fêmeas foram infecundas. Por meio do teste do $\chi^{2}$ verificouse que a diferença na fecundidade das fêmeas criadas nas duas dietas não é significativo $(6,34)$ quando $\alpha=$
0,01; porém este tem significância quando se considera $\alpha=0,1$. Biologicamente, esta diferença é significante visto que o número de descendentes oriundos das fêmeas criadas com farinha de carne é quase o dobro das criadas em peixe in natura. Segundo Roper et al. (1996), oaumento do tempo de desenvolvimentolarval, verificado na farinha de carne, pode ocorrer por atraso dos indivíduos na obtenção do peso mínimo necessário para pupariação.

É relevante o reconhecimento da efetividade de uma dieta artificial baseado no potencial reprodutivo do objeto de estudo, pois, em alguns casos, a falta de micronutrientes pode afetar a produção de ovos sem comprometer a massa corporal e o tamanho do inseto (Colegrave, 1993). Porém, no presente estudo, verificou-se que a infecundidade está diretamente relacionada ao peso das fêmeas. O peso médio das fêmeas infecundas, alimentadas com ambas as dietas durante sua fase larval não, variou muito 47,611 mg $\pm 10,908$ e $47,545 \mathrm{mg} \pm 10,234$ para o meio de peixe e de farinha de carne, respectivamente. Quando comparados estes valores com a média do peso das fêmeas fecundas $(65,185 \mathrm{mg} \pm 10,285$ e $64,882 \mathrm{mg} \pm 9,166)$, constatou-se que o peso médio das infecundas mostrou-se bastante inferior, em ambas as dietas testadas. Estes resultados foram comprovados pelo teste do $\chi^{2}$, pois o peso influi na fecundidade e no desempenho reprodutivo.

Estudos anteriores demonstraram que, dentre diversos meios testados, o peixe apresenta maior atratividade para que fêmeas de C. megacephala efetuem suas posturas (D'AlmeIdA; S AlviANo, 1996; SILvAet al., 2005). Para tal, a escolha do peixe como dieta natural para criação desta espécie em laboratório baseou-se nos hábitos naturais que esta possui em sua região de origem. Porém, este estudo demonstrou que uma dieta artificial à base de farinha de carne é viável, senão mais vantajosa, do que aquela que supostamente reproduz as condições naturais ideais ao desenvolvimento desta espécie. Tal fato pode ser justificado, pois $C$. megacephala é uma espécie com grande plasticidade e ao ser introduzida neste continente modificou seus hábitos, visitando também carcaças de mamíferos e vísceras de diversos animais. Segundo PrADO; GuIMARÃEs (1982), os hábitos alimentares generalistas de C. megacephala possibilitam sua adaptação em variados ambientes. Para DiDHAM et al. (1996), as espécies generalistas e oportunistas respondem melhor às mudanças ambientais do que as especialistas.

Alguns autores (MendonçA; D'Almeida, 2004; D'AlMEIDA; Lopes, 1983; LEAL et al., 1982) salientaram a preferência e os bons resultados obtidos com califorídeos criados em carne em laboratório, porém, a utilização de dietas artificiais eficientes minimizam os problemas quanto a estocagem, putrefação e ônus econômicos. 
Tabela 4 - Influência do meio de cultura sobre o período e a viabilidade de larvas e pupas deChrysomya megacephala, em laboratório.

\begin{tabular}{lccc}
\hline & \multicolumn{2}{c}{ Período } & \\
\cline { 2 - 4 } Fase (meio de cultura) & Média (dias) & Variação (dias) & Viabilidade (\%) \\
\hline Larva (peixe) & 3,514 & $3-4$ & 95 \\
Larva (farinha de carne) & 3,557 & $3-6$ & 91,5 \\
Pupa (peixe) & 4,537 & $4-5$ & 83,3 \\
Pupa (farinha de carne) & 6,43 & $4-7$ & 86 \\
\hline
\end{tabular}

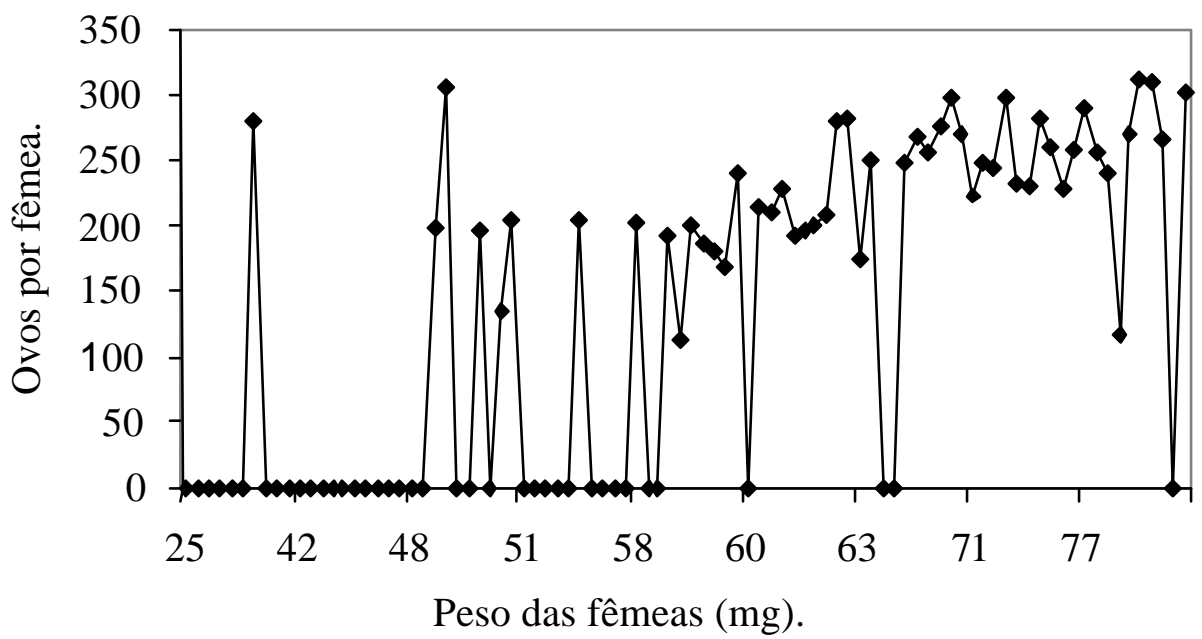

Fig. 1 - Relação entre peso e número de ovos por fêmea de Chrysomya megacephalacriada durante sua fase larval em peixe in natura, em laboratório.

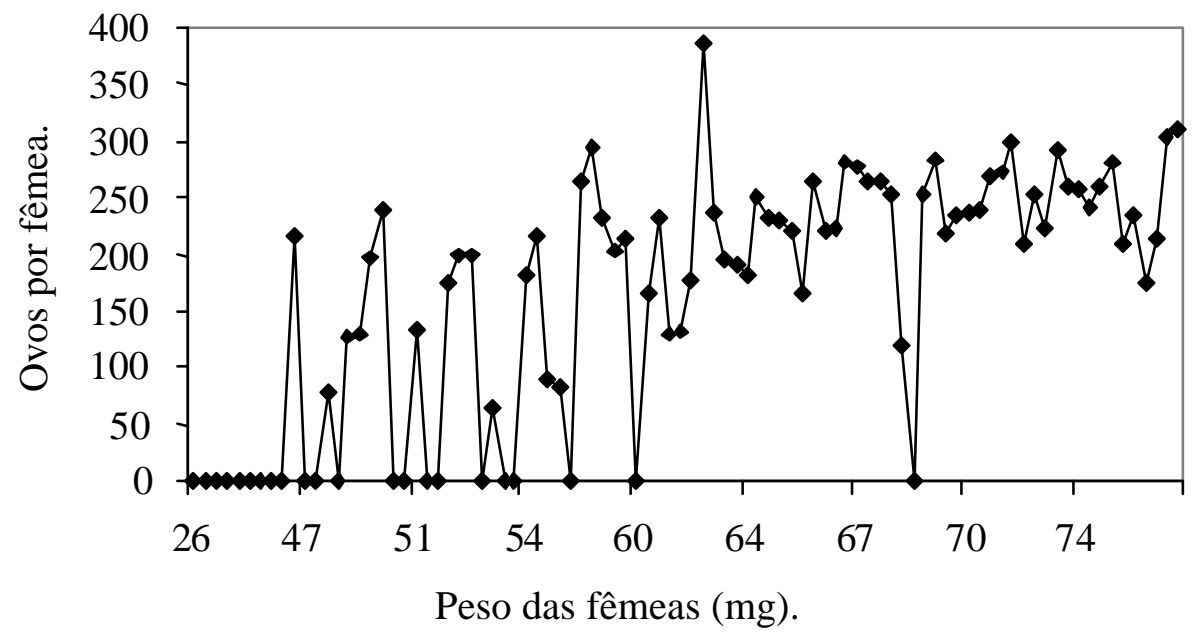

Fig. 2 - Relação entre peso e número de ovos por fêmea deChrysomya megacephalacriada durante sua fase larval em farinha de carne, em laboratório.

\section{CONCLUSÕES}

- Odesenvolvimentoe a performance reprodutiva das fêmeas adultas de C. megacephala são influenciadas pela dieta utilizada durante sua fase larval.

- O peso das fêmeas adultas influenciou a fecundidade, tanto na dieta de farinha de carne como na de peixe in natura.
- Larvas de C. megacephala, alimentadas com farinha decarne, originaram maior número de fêmeas fecundas quando comparada com aquelas que se alimentam da dieta de peixe in natura.

- Considerando a adaptação deC. megacephalaàs nossas condições, conclui-se que uma dieta artificial à base de farinha de carneé vantajosa quand o comparada a uma dieta constituída por peixe in natura. 


\section{REFERENNCIAS}

BARBOSA, L.S.; JESUS, D.M.L. de; COELHO, V.M.A. Longevidade e capacidade reprodutiva de casais de Chrysomya megacephala (Diptera, Calliphoridae) oriundos de larvas criadas em dieta natural e oligolítica. Revista Brasileira de Zoociências, v.6, n.2, p.207-217, 2004.

COLEGRAVE, N. Does larval competition effect fecundity independently of its effects on adult weight? Ecological Entomology, v.18, p.275-277, 1993.

COLLINS, N.C. Developmental responses to food limitation as indicators of environmental conditions for Ephydra cinerea Jones (Diptera). Ecology, v.61, p.650$661,1980$.

COSTA, P.R.P.; WIEGAND, M.M.; BRUM, J.G.W.; RIBEIRO, P.B. Flutuação populacional das espécies de Chrysomya (Diptera: Calliphoridae) no município do Capão do Leão, RS. Arquivo Brasileiro de Medicina Veterinária e Zootecnia, v.44, n.4, p.289-296, 1992.

D'ALMEIDA, J.M.; LOPES, H.S. Sinantropia de dípteros muscóides (Calliphoridae) no Estado do Rio de Janeiro. Arquivos da Universidade Federal Rural do Rio de Janeiro, v.6, n.1, p.39-48, 1983.

D'ALMEIDA, J.M.; SALVIANO, R.J.B. Feeding preference of the larvae of Chrysomya megacephala (Fabricius) (Diptera: Calliphoridae) and Ravinia belforti (Prado e Fonseca) (Diptera: Sarcophagidae) concerning different diets. Memórias do Instituto Oswaldo Cruz, v.91, n.1, p.137-138, 1996.

DIDHAM, R.K.; GHAZOUL, J.; STORK, N.E.; DAVIS, A J. Insects in fragmented forests: a functional approach. Trends in Ecology and Evolution, v.11, p.255-260, 1996.

ESSER, J.R. Factors influencing oviposition, larval grow and mortality in Chrysomya megacephala (Diptera: Calliphoridae), a pest of salted dried fish in southeast Ásia. Bulletin of Entomological Research, v.80, p.369-376, 1990.

GOODBROD, J.R.; GOFF, M.L. Effects of larval population density on rates of development and interactions between two species of Chrysomya (Diptera: Calliphoridae) in laboratory culture. Journal of Medical Entomology, v.27, p.338-343, 1990.

GUIMARÃES, J.H.; PRADO, A.P.; LINHARES, A.X. Three newly introduced blow fly species in southern Brazil (Diptera: Calliphoridae). Revista Brasileira de Entomologia, v.22, p.53-60, 1978.

KAMAL, A.S. Comparative study of thrirteen species of sarcosaprophagous Calliphoridae and

Sarcophagidae (Diptera): I. Bionomics. Annals of the Entomological Society of America, v.51, p.261-270, 1958.
LEAL, T.T.; PRADO, A.P.; ANTUNES, J.A. Rearing the larvae of the blowfly Chrysomya cloropyga (Wiedmann) (Diptera: Calliphoridae) on oligidic diets. Revista Brasileira de Zoologia, v.1, p.41-44, 1982.

MENDONÇA, P.M.M.; D'ALMEIDA, J.M. Desenvolvimento pós-embrionário de Chrysomya megacephala (Fabricius, 1794) (Diptera: Calliphoridae) em dietas artificiais à base de leite. Entomologia y Vectores, v.11, n.1, p.59-67, 2004.

OLIVEIRA, C.M.B. Ocorrência e flutuação populacional de três espécies do gênero Chrysomya. Pesquisa Agropecuária Brasileira, v.30, n.4, p.497-498, 1982.

PARRA, J.R.P. Consumo e utilização de alimentos por insetos. In: PANIZZI, A.R.; PARRA, J.R.P. (Ed.). Ecologia nutricional de insetos e suas implicações no manejo de pragas. São Paulo: Manole, 1990. p.9-57.

PRADO, A.P.; GUIMARÃES, J.H. Estado atual de propagação e distribuição do gênero Chrysomya Robineau-Desvoidy na Região Neotropical (Diptera: Calliphoridae). Revista Brasileira de Entomologia, v.26, n.3/4, p.225-231, 1982.

PUTMAN, R.J. Dynamics of the blowfly, Calliphora erythrocephala, within carrion. Journal of Animal Ecology, v.46, p.853-866, 1977.

REIS, S.F.; STANGENHAUS, G.; GODOY, W.A.C.; von ZUBEN, C.J.; RIBEIRO, O.B. Variação dos caracteres bionômicos em função de densidade larval em Chrysomya megacephala (Diptera: Calliphoridae). Revista Brasileira de Entomologia, v.38, p.33-46, 1994.

REIS, S.F.; TEIXEIRA, M.A.; von ZUBEN, F.J.; GODOY, W.A.C.; von ZUBEN, C.J. Theoretical dynamics of experimental populations of introduced and native blowflies. Journal of Medical Entomology, v.33, p.537-544, 1996.

ROPER, C.; PIGNATELLI, P.; PARTRIDGE, L. Evolutionary responses of Drosophila melanogaster life history to differences in larval density. Journal of Evolutionary Biology, v.9, p.609-622, 1996.

SILVA, A.S.; HECK, C.A.; DOYLE, R.L.; MONTEIRO, S.G. Levantamento das espécies de dípteros na região de Santa Maria baseado em diferentes substratos. Revista da Faculdade Zootecnia, Veterinária e Agronomia, v.12, n.1, p.51-58, 2005.

SILVEIRA NETO, S.; NAKANO, O.; BARBIN, D.; VILLA NOVA, N. A.. Manual de Ecologia de Insetos. São Paulo: Agronômica Ceres, 1976. 419p.

STATISTIX 8. Statistix User's Manual. Analytical software. Tllahassee, FL. 2003.

TARDELLI, C.A.; GODOY, W.A.C.; MANCERA, P.F.A. Population dynamics of Musca domestica (Diptera: 
Muscidae): Experimental and theoretical studies at different temperatures. Brazilian Archives of Biology and Technology, v.47, n.5, p.775-783, 2004.

vON ZUBEN, C.J. Competição larval em Chrysomya megacephala (Diptera: Calliphoridae): estimativas de perdas em biomassa e na fecundidade e cálculo da conversão de alimento em biomassa. Revista Brasileira de Entomologia, v.37, n.4, p.793-802, 1993.

VON ZUBEN, C.J.; BASSANEZI, R.C.; REIS, S.F.; GODOY, W.A.C.; von ZUBEN, F.J. Theoretical approaches to forensic entomology: I. Mathematical model of postfeeding larval dispersal. Journal of Applied Entomology, v.120, p.379-382, 1996.
vON ZUBEN, C.J; STANGENHAUS, G.; GODOY, W.A.C. Competição larval em Chrysomya megacephala (F.) (Diptera: Calliphoridae): efeitos de diferentes níveis de agregação larval sobre estimativas de peso, fecundidade e investimento reprodutivo. Revista Brasileira de Biologia, v.60, n.2, p.195-203, 2000.

ZIMMER, C.R.; PIRES, S.M.; CÁRCAMO, M.C. RIBEIRO, P.B. Efeitos da competição larval intra-específica sobre caracteres biométricos de Muscina stabulans (Fallén, 1817) (Diptera: Muscidae) em laboratório. Arquivos do Instituto Biológico, São Paulo, v.73, n.2, p.203-209, 2006.

Recebido em 28/4/07

Aceito em 4/12/08 